\section{Übergewicht und Asthma: Suche nach gemeinsamen Genen}

\author{
Epidemiologische Studien weisen konsistent auf eine Assoziation von \\ Übergewicht und Asthma hin. In einer Genotypisierungsstudie wurde \\ daher nach gemeinsamen Genvarianten gesucht.
}

$\mathrm{n}$ einer Datenbankrecherche wurden zunächst genomweite Assoziationsstudien (GWAS) und Kandidatengenstudien ermittelt, die sich auf den BodyMass-Index (BMI) und Asthma bezogen. Die darin identifizierten Gene wurden mit Daten des Childhood Asthma Management Program (CAMP) abge-

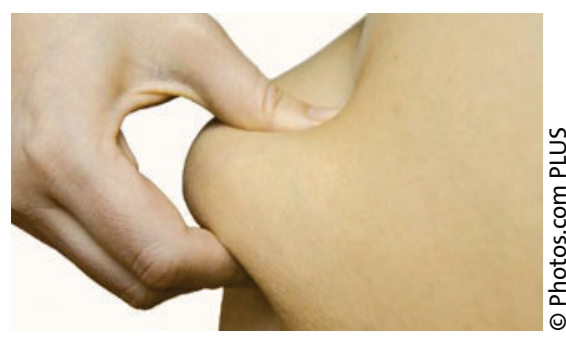

Ob ein erhöhter Body-Mass-Index Rückschlüsse auf ein genetisch bedingtes Asthmarisiko zulässt, sollte eine Genotypisierungsstudie zeigen. glichen, um mögliche Assoziationen zwischen Single-Nucleotid-Polymorphismen (SNP) in den Genen und dem Vorliegen von Asthma und erhöhtem BMI in einer Kohorte von Kindern zu ermitteln. CAMP war eine multizentrische Studie mit 1.041 asthmakranken Kindern, von denen größtenteils DNA-Proben vorlagen. Ein Drittel der Kinder war übergewichtig.

Einer der in den genomweiten Assoziationsstudien signifikant mit Übergewicht korrelierten Polymorphismen ( $\mathrm{p}=$ $\left.4 \times 10^{-4}\right)$, rs10938397 nahe dem Genabschnitt für Glucosamin-6-PhosphatDeaminase 2 (GNPDA2), war auch mit Asthma ( $\mathrm{p}=0,03)$ assoziiert. Von den signifikant mit Asthma korrelierten Polymorphismen aus Assoziationsstudien und Kandidatengenstudien war keiner mit einem erhöhten BMI assoziiert. Bei einer Analyse aller verfügbaren Polymorphismen in den identifizierten Genen wurden jedoch eine Reihe weiterer Gene gefunden, die nominell mit beiden Phänotypen assoziiert waren. Dazu gehörte unter den in genomweiten Assoziationsstudien mit Übergewicht korrelierten Genen dasjenige für das „roundabout, axon guidance receptor, homolog 1 “ $(R O B O 1)$ und unter den mit Asthma korrelierten Genen dasjenige für den Protein-Tyrosin-Phosphatase RezeptorTyp D (PTPRD). Das Gen der ProteinKinase $\mathrm{C} \alpha(P R K C A)$ war sowohl mit Übergewichts- als auch mit AsthmaKandidatengenen assoziiert.

Fazit: Es gibt einige bekannte Gene, deren Polymorphismen sowohl mit Übergewicht als auch mit Asthma - wenngleich nicht signifikant - assoziiert sind. Wahrscheinlich existieren noch weitere, bisher nicht entdeckte Loci für gemeinsame genetische Determinanten beider Phänotypen. Dr. Barbara Kreutzkamp

Melén E et al. Analyses of shared genetic factors between asthma and obesity in children. J Allergy Clin Immunol 2010; 126: 631-7

hatten ein erhöhtes Risiko für ein neu auftretendes atopisches Asthma (aMOR 4,1; 95\%-CI 1,9 bis 8,8), eine Rhinitis im Kindesalter war ein Risikofaktor für die Persistenz des kindlichen Asthmas als atopisches Asthma des Erwachsenen (aMOR 2,7; 95\%-CI 1,3 bis 5,6). 29,7\% des persistierenden und $18,1 \%$ des neu auftretenden atopischen Asthmas dürfte auf der Basis von Ekzemen und Rhinitis in der Kindheit entstanden sein.

Fazit: Ekzem und/oder Rhinitis in der Kindheit sind starke Prädiktoren für atopisches Asthma im Erwachsenenalter. Beide sollten deshalb konsequent behandelt werden, um den atopischen Marsch aufzuhalten. Dr. Barbara Kreutzkamp

Martin PE et al. Childhood eczema and rhinitis predict atopic but not nonatopic adult asthma: A prospective cohort study over 4 decades. J Allergy Clin Immunol 2011 Mar31 [Epub ahead of print] 